\title{
Divisorial Valuations via Arcs
}

\author{
By \\ Tommaso de FerneX*, Lawrence EIN** and Shihoko IsHII***
}

\begin{abstract}
This paper shows a finiteness property of a divisorial valuation in terms of arcs. First we show that every divisorial valuation over an algebraic variety corresponds to an irreducible closed subset of the arc space. Then we define the codimension for this subset and give a formula of the codimension in terms of "relative Mather canonical class". By using this subset, we prove that a divisorial valuation is determined by assigning the values of finite functions. We also have a criterion for a divisorial valuation to be a monomial valuation by assigning the values of finite functions.
\end{abstract}

\section{Introduction}

Let $X$ be a complex algebraic variety of dimension $n \geq 1$. An important class of valuations of the function field $\mathbb{C}(X)$ of $X$ consists of divisorial valuations. These are valuations of the form

$$
v=q \operatorname{val}_{E}: \mathbb{C}(X)^{*} \longrightarrow \mathbb{Z}
$$

where $E$ is a prime divisor on a normal variety $Y$ equipped with a proper, birational morphism $f: Y \rightarrow X, q=q(v)$ is a positive integer number called the multiplicity of $v$, and for every $h \in \mathbb{C}(X)^{*}$ that is regular at the generic point of $f(E), \operatorname{val}_{E}(h):=\operatorname{ord}_{E}(h \circ f)$ is the order of vanishing of $h \circ f$ at

Communicated by S. Mukai. Received January 30, 2007. Revised September 14, 2007.

2000 Mathematics Subject Classification(s): 14J17, 14M99.

*Department of Mathematics, University of Utah, 155 South 1400 East, Salt Lake City, UT 48112-0090, USA.

e-mail: defernex@math.utah.edu

** Department of Mathematics, University of Illinois at Chicago, 851 S. Morgan St., M/C. 249, Chicago, IL 60607-7045, USA.

e-mail: ein@math.uic.edu

*** Department of Mathematics, Tokyo Institute of Technology, Oh-Okayama, Meguro, 1528551, Tokyo, Japan.

e-mail: shihoko@math.titech.ac.jp

(c) 2008 Research Institute for Mathematical Sciences, Kyoto University. All rights reserved. 
the generic point of $E$. It is a theorem of Zariski that the set of valuation rings associated with this class of valuations coincides with the set of discrete valuation $\operatorname{rings}\left(R, \mathfrak{m}_{R}\right)$ of $\mathbb{C}(X)$ with $\operatorname{trdeg}\left(R / \mathfrak{m}_{R}: \mathbb{C}\right)=n-1$. Thanks to Hironaka's resolution of singularities, divisorial valuations had acquired a fundamental role in singularity theory.

Since the publication of the influential paper of Nash $[\mathrm{N}]$ and the introduction of motivic integration (see, e.g., [K], [DL], [B]), it become apparent the close link between certain invariants of singularities related to divisorial valuations and the geometry of arc spaces. This link was first explored by Mustaţă in [M1], [M2], and then further studied in [ELM], [I2], [I3]. In particular, when the ambient variety $X$ is smooth, it is shown in [ELM] how one can reinterpret invariants such as multiplier ideals in terms of properties of certain subsets in the space of $\operatorname{arcs} X_{\infty}$ of $X$.

The main purpose of this paper is to extend the results of [ELM] to arbitrary varieties and to employ such results towards the characterization of divisorial valuations by evaluation against finite numbers of functions.

Naturally associated to the valuation $v=q \operatorname{val}_{E}$, there is a subset

$$
W(v)=W(E, q) \subset X_{\infty}
$$

constructed as follows. We can assume without loss of generality that both $Y$ and $E$ are smooth. Then $W(v)$ is defined as the closure of the image, via the natural map $Y_{\infty} \rightarrow X_{\infty}$, of the set of arcs on $Y$ with order of contact along $E$ equal to $q$. It turns out that

$$
v=\operatorname{val}_{W(v)},
$$

where for every irreducible constructible subset $C \subset X_{\infty}$ that is not contained in the arc space of any proper closed subvariety of $X$, we define a valuation $\operatorname{val}_{C}: \mathbb{C}(X)^{*} \rightarrow \mathbb{Z}$ by taking the order of vanishing (or polarity) along the generic point of $C$ (see Definition 2.3). Although the subsets of $X_{\infty}$ of the form $W(v)$ are quite special, there is a much larger class of subsets $C \subseteq X_{\infty}$, such that the associated valuation $\mathrm{val}_{C}$ is a divisorial valuation. These sets are called divisorial sets. It was shown in [I3] that sets of the form $W(v)$ are maximal (with respect to inclusion) among all divisorial sets defining the same valuation; for this reason they are called maximal divisorial sets.

Other important classes of subsets of $X_{\infty}$ are those consisting, respectively, of (quasi)-cylinders and contact loci. The valuations associated to irreducible components of such sets are called cylinder valuations and contact valuations, respectively. As it turns out, maximal divisorial sets belong to these classes of sets. Generalizing to singular varieties some results of $[\mathrm{ELM}]$, we obtain the 
following properties:

(a) every divisorial valuation is a cylinder valuation (Theorem 3.9);

(b) every cylinder valuation is a contact valuation (Proposition 3.10);

(c) every contact valuation is a divisorial valuation (Proposition 2.12).

Geometrically, the correspondence between divisorial valuations and cylinder valuations is constructed by associating to any divisorial valuation $v$ the subset $W(v) \subset X_{\infty}$. The fact that $W(v)$ is a quasi-cylinder shows that $v$, being equal to $\operatorname{val}_{W(v)}$, is a cylinder valuation. The other direction of the correspondence is more elaborate: starting from a cylinder valuation val $_{C}$, one first realizes $\mathrm{val}_{C}$ as a contact valuation, that is, a valuation determined by an irreducible component of a contact locus, the definition of which involves certain conditions on the order of contact along some subscheme of $X$. The divisor $E$ is then extracted by a suitable weighted blowup on a log resolution of this subscheme, and $q$ is determined by the numerics involved in the construction.

A key point in the proof of these assertions, as well as a fundamental property for many applications, is a codimension formula for the maximal divisorial set $W(v)$ associated to a divisorial valuation $v$. When $X$ is a smooth variety, it was shown in $[\mathrm{ELM}]$ that

$$
\operatorname{codim}\left(W(v), X_{\infty}\right)=k_{v}(X)+q(v),
$$

where $k_{v}(X):=v\left(K_{Y / X}\right)$ is the discrepancy of $X$ along $v$ and $q(v)$ is the multiplicity of $v$. The definition of discrepancy needs to be modified when $X$ is singular. Given an arbitrary variety $X$, we take a resolution of singularities $f: Y \rightarrow X$ that factors through the Nash blowup

$$
\nu: \widehat{X} \longrightarrow X
$$

and define the relative Mather canonical divisor $\widehat{K}_{Y / X}$ of $f$ (see Definition 1.1). This divisor, which is defined in total generality, is always an effective integral divisor, and it coincides with $K_{Y / X}$ when $X$ is smooth (the two divisors are in general different for $\mathbb{Q}$-Gorenstein varieties). The relative Mather canonical divisor plays a fundamental role in the geometry of arc spaces and the changeof-variables formula in motivic integration. Defining $\widehat{k}_{v}(X):=v\left(\widehat{K}_{Y / X}\right)$, the codimension formula of $[\mathrm{ELM}]$ generalizes to arbitrary varieties as follows.

Theorem 0.1. With the above notation, we have

$$
\operatorname{codim}\left(W(v), X_{\infty}\right)=\widehat{k}_{v}(X)+q(v) .
$$


Using the interpretation of divisorial valuations via arc spaces, we show that each divisorial valuation $v$ is characterized by its values $v\left(f_{i}\right)=v_{i}$ on a finite number of functions $f_{i}$. More precisely, we have the following result.

Theorem 0.2. Suppose that $X=\operatorname{Spec} A$ is an affine variety, and let $v$ be a divisorial valuation over $X$. Then there exists elements $f_{1}, \ldots, f_{r} \in A$ and $v_{1}, \ldots, v_{r} \in \mathbb{N}$ such that for every $f \in A \backslash\{0\}$

$$
v(f)=\min \left\{v^{\prime}(f) \mid v^{\prime} \text { is a divisorial valuation such that } v^{\prime}\left(f_{i}\right)=v_{i}\right\} .
$$

Theorem 0.2 is obtained by determining functions $f_{i}$ and numbers $v_{i}$ such that

$$
W(v) \subset \bigcap_{i=1}^{r} \operatorname{Cont}^{v_{i}}\left(f_{i}\right),
$$

with equality holding off a set contained in $(\operatorname{Sing} X)_{\infty}$. This is the content of Theorem 4.2. A similar result can be obtained using MacLane's results from [ML] (see Remark 4.6); it would be interesting to further investigate the connection between MacLane's key polynomials and the functions $f_{i}$ determined in the proof of the above results, and to study properties that the first ones satisfy with respect to the geometry of arc spaces.

In the case of monomial valuations on toric varieties, we apply Theorem 0.1 to give a precise characterization in terms of a system of parameters. For simplicity, we present here the result in the special case when the toric variety is equal to $\mathbb{C}^{n}$.

Theorem 0.3. Let $v$ be a divisorial valuation of $\mathbb{C}\left(x_{1}, \ldots, x_{n}\right)$, centered at the origin 0 of $X=\mathbb{C}^{n}$. Assume that there are positive integers $a_{1}, \ldots, a_{n}$ such that

$$
v\left(x_{i}\right) \geq a_{i} \quad \text { and } \quad \sum a_{i} \geq k_{v}(X)+q(v) .
$$

Then $v$ is a monomial valuation determined by the weights $a_{i}$ assigned along the parameters $x_{i}, q(v)=\operatorname{gcd}\left(a_{1}, \ldots, a_{n}\right)$, and equalities hold in both formulas in $(2)$.

The more general version of this result, holding for arbitrary singular toric varieties, requires some additional notation, and is given in Theorem 5.1.

The authors express their hearty thanks to Bernard Teissier for his helpful advice, and would like to thank Mircea Mustaţă for many helpful discussions, in particular about the proof of Theorem 5.1. 


\section{§1. The Relative Mather Canonical Divisor}

In this preliminary section, we review the construction of the Nash blowup and define a generalization of the relative canonical divisor to certain resolutions of arbitrarily singular varieties. This will give a geometric interpretation of a certain ideal sheaf that governs the dimension of fibers of maps between arc space, and consequently the change-of-variable formula in motivic integration.

We start with an arbitrary complex variety $X$ of dimension $n$. Note that the projection

$$
\pi: \mathbb{P}_{X}\left(\wedge^{n} \Omega_{X}\right) \longrightarrow X
$$

is an isomorphism over the smooth locus $X_{\text {reg }} \subseteq X$. In particular, we have a section $\sigma: X_{\text {reg }} \rightarrow \mathbb{P}_{X}\left(\wedge^{n} \Omega_{X}\right)$.

Definition 1.1. The closure of the image of the section $\sigma$ is the Nash blowup of $X$, and is denoted by $\widehat{X}$ :

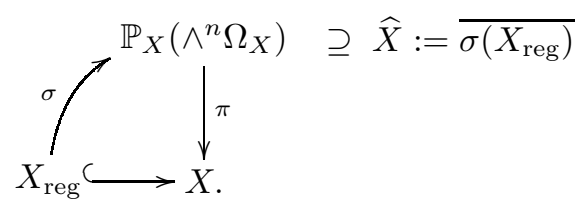

The Nash blowup $\widehat{X}$ comes equipped with the morphism

$$
\nu:=\left.\pi\right|_{\widehat{X}}: \widehat{X} \longrightarrow X
$$

and the line bundle

$$
\widehat{K}_{X}:=\left.\mathcal{O}_{\mathbb{P}_{X}\left(\wedge^{n} \Omega_{X}\right)}(1)\right|_{\widehat{X}} .
$$

We call this bundle the Mather canonical line bundle of $X$.

Remark 1.2. If $X$ is smooth, then $\widehat{X}=X$ and $\widehat{K}_{X}$ is just the canonical line bundle of $X$.

The original definition of Nash blowup is slightly different. Assuming the existence of an embedding $X \hookrightarrow M$ into a manifold $M$, one can consider the Grassmann bundle $G\left(\Omega_{M}, n\right)$ over $M$ of rank $n$ locally free quotients sheaves of $\Omega_{M}$. The map

$$
x \mapsto\left(\left(\Omega_{X}\right)_{x} \nleftarrow\left(\Omega_{M}\right)_{x}\right) \in G\left(\Omega_{M}, n\right)_{x},
$$

defined for every smooth point $x$ of $X$, gives a section $\widetilde{\sigma}: X_{\text {reg }} \rightarrow G\left(\Omega_{M}, n\right)$. Then one takes the closure of the image of this section in $G\left(\Omega_{M}, n\right)$. As we prove next, the two constructions agree. 
Proposition 1.3. Keeping the above notation, let $\widetilde{X}$ denote the closure of $\widetilde{\sigma}\left(X_{\mathrm{reg}}\right)$ in $G\left(\Omega_{M}, n\right)$, and let $\widetilde{\nu}: \widetilde{X} \rightarrow X$ the induced morphism. Then $\widetilde{X}=\widehat{X}$ and $\widetilde{\nu}=\nu$.

Proof. We have exact sequences



where $\left.\Omega_{M}\right|_{\widetilde{X}}$ is $\left.\varpi^{*} \Omega_{M}\right|_{\widetilde{X}}$ for the projection $\varpi: G\left(\Omega_{M}, n\right) \rightarrow M$ and it also coincides with $\widetilde{\nu}^{*}\left(\left.\Omega_{M}\right|_{X}\right)$. The top row is the restriction of the universal sequence of the Grassmann bundle $G\left(\Omega_{M}, n\right)$, and the second is the pull back of the sequence of differentials determined by the inclusion of $X$ in $M$. Over the smooth locus of $X$ we have

$$
\left.S\right|_{\widetilde{\sigma}\left(X_{\text {reg }}\right)}=\left.\operatorname{ker}(q)\right|_{\widetilde{\sigma}\left(X_{\text {reg }}\right)} .
$$

Then, since $\operatorname{ker}(q)$ is torsion free and the top sequence has a local splitting, the inclusion $j$ factors through $i$ and an inclusion $\left.\operatorname{ker}(q) \hookrightarrow S\right|_{\widetilde{X}}$. Therefore $p$ factors through $q$ and a surjection $\left.\widetilde{\nu}^{*} \Omega_{X} \rightarrow Q\right|_{\widetilde{X}}$. After taking wedges, we obtain a commutative diagram of surjections

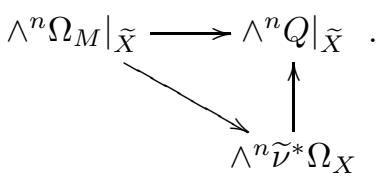

Now we consider the inclusion over $\widetilde{X}$

$$
G\left(\left.\Omega_{M}\right|_{\tilde{X}}, n\right) \hookrightarrow \mathbb{P}_{\widetilde{X}}\left(\left.\wedge^{n} \Omega_{M}\right|_{\tilde{X}}\right)
$$

given by Plücker embedding. The factorization (3) implies that the image of $\widetilde{X}$ under this embedding is contained in $\mathbb{P}_{\widetilde{X}}\left(\wedge^{n} \widetilde{\nu}^{*} \Omega_{X}\right)$, when the latter is viewed as a subvariety of $\mathbb{P}_{\widetilde{X}}\left(\left.\wedge^{n} \Omega_{M}\right|_{\widetilde{X}}\right)$ via the natural embedding. Therefore, by the compatibility of Plücker embeddings, the image of $\widetilde{X}$ under the embedding

$$
G\left(\left.\Omega_{M}\right|_{X}, n\right) \hookrightarrow \mathbb{P}_{X}\left(\left.\wedge^{n} \Omega_{M}\right|_{X}\right)
$$

is contained in $\mathbb{P}_{X}\left(\wedge^{n} \Omega_{X}\right)$. Then, restricting over the regular locus of $X$, we have

$$
\tilde{X} \cap \pi^{-1}\left(X_{\text {reg }}\right)=\pi^{-1}\left(X_{\text {reg }}\right)=\widehat{X} \cap \pi^{-1}\left(X_{\text {reg }}\right) .
$$


Since $\widetilde{X}$ and $\widehat{X}$ are irreducible varieties, both surjecting onto $X$, we conclude that $\widetilde{X}=\widehat{X}$. The equality $\widetilde{\nu}=\nu$ follows by the fact that the construction is carried over $X$.

Remark 1.4. The original construction of the Nash blowup using Grassmann bundles can be given without using (or assuming) any embedding, by considering the Grassmann bundle $G\left(\Omega_{X}, n\right)$ on $X$ of rank $n$ locally free quotient sheaves of $\Omega_{X}$. Notice that $G\left(\Omega_{X}, n\right)$ and $\mathbb{P}_{X}\left(\wedge^{n} \Omega_{X}\right)$ agree over the smooth locus of $X$.

Remark 1.5. Mather used the above construction to propose a generalization to singular varieties of the notion of Chern classes of manifolds, by considering the class $\widetilde{\nu}_{*}\left(c\left(\left.Q^{*}\right|_{\widetilde{X}}\right) \cap[\widetilde{X}]\right)$ in $A_{*}(X)$. This is known as the MatherChern class of $X$. The push-forward $\nu_{*}\left(c_{1}\left(\widehat{K}_{X}^{-1}\right) \cap[\widehat{X}]\right)$ is equal to the first Mather-Chern class of $X$.

Now consider any resolution of singularities $f: Y \rightarrow X$ factoring through the Nash blowup of $X$, so that we have a commutative diagram



Definition 1.6. Let $\widehat{K}_{Y / X}$ be the divisor supported on the exceptional divisor on $Y$ and linearly equivalent to $K_{Y}-\widehat{f}^{*} \widehat{K}_{X}$. We call it the relative Mather canonical divisor of $f$.

Proposition 1.7. The relative Mather canonical divisor $\widehat{K}_{Y / X}$ is an effective divisor and satisfies the relation:

$$
d f\left(f^{*} \wedge^{n} \Omega_{X}\right)=\mathcal{O}_{Y}\left(-\widehat{K}_{Y / X}\right) \cdot \wedge^{n} \Omega_{Y},
$$

where $d f: f^{*} \wedge^{n} \Omega_{X} \rightarrow \wedge^{n} \Omega_{Y}$ is the canonical homomorphism.

Proof. By generic smoothness of $X$, the kernel of the morphism $\nu^{*} \wedge^{n} \Omega_{X} \rightarrow \widehat{K}_{X}$ is torsion, hence, pulling back to $Y$, we obtain a commutative diagram

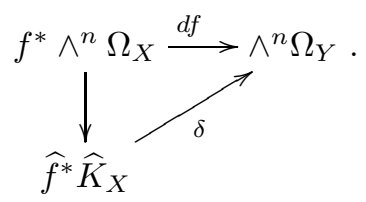


Then, we have an effective divisor $D$ with the support on the exceptional divisor such that

$$
\operatorname{im}(\delta)=\mathcal{O}_{Y}(-D) \cdot \wedge^{n} \Omega_{Y} .
$$

It follows that $D$ is linearly equivalent to $K_{Y}-\widehat{f}^{*} \widehat{K}_{X}$, therefore we obtain $D=\widehat{K}_{Y / X}$.

For the second statement, we should note that $\nu^{*} \wedge^{n} \Omega_{X} \rightarrow \widehat{K}_{X}$ is surjective as $\widehat{K}_{X}$ is relatively very ample with respect to $\pi: \mathbb{P}_{X}\left(\wedge^{n} \Omega_{X}\right) \rightarrow X$. This gives the surjectivity of $f^{*} \wedge^{n} \Omega_{X} \rightarrow \widehat{f}^{*} \widehat{K}_{X}$ and the second statement.

Note that $\widehat{K}_{Y / X}$ is always an effective integral Cartier divisor, and in particular it is in general different from the relative canonical divisor defined in the $\mathbb{Q}$-Gorenstein case. In fact, the following property holds.

Proposition 1.8. Let $X$ be a normal and locally complete intersection variety, and let $f: Y \rightarrow X$ be a resolution of singularities factoring through the Nash blowup of $X$. Then $\widehat{K}_{Y / X}=K_{Y / X}$ if and only if $X$ is smooth.

Proof. It follows by [EM1] that the difference $\widehat{K}_{Y / X}-K_{Y / X}$ is given by the vanishing order of the Jacobian ideal sheaf of $X$.

Definition 1.9. For every prime divisor $E$ on $Y$, we define

$$
\widehat{k}_{E}(X):=\operatorname{ord}_{E}\left(\widehat{K}_{Y / X}\right)
$$

and call it the Mather discrepancy of $X$ along $E$. More generally, if $v$ is a divisorial valuation over $X$, then we can assume without loss of generality that $v=q \operatorname{val}_{E}$ for a prime divisor $E$ on $Y$ and a positive integer $q$, and define the Mather discrepancy of $X$ along $v$ to be

$$
\widehat{k}_{v}(X):=q \cdot \widehat{k}_{E}(X) .
$$

If $X$ is smooth, then we denote $k_{E}(X):=\widehat{k}_{E}(X)$ and $k_{v}(X):=\widehat{k}_{v}(X)$.

\section{§2. Contact Loci in Arc Spaces and Valuations}

In this section we set up basic statements for contact loci and divisorial valuations.

Definition 2.1. $\quad$ Let $X$ be a scheme of finite type over $\mathbb{C}$ and $K \supset \mathbb{C}$ a field extension. A morphism $\alpha: \operatorname{Spec} K[[t]] \rightarrow X$ is called an arc of $X$. We denote the closed point of Spec $K[[t]]$ by 0 and the generic point by $\eta$. For $m \in \mathbb{N}$, a morphism $\beta: \operatorname{Spec} K[t] /\left(t^{m+1}\right) \rightarrow X$ is called an $m$-jet of $X$. Denote the space of arcs of $X$ by $X_{\infty}$ and the space of $m$-jets of $X$ by $X_{m}$. See [M2] for more details. 
The concept "thin" in the following is first introduced in [ELM] and a "fat arc" is introduced and studied in [I2].

Definition 2.2. $\quad$ Let $X$ be a variety over $\mathbb{C}$. We say that an arc $\alpha:$ Spec $K[[t]] \rightarrow X$ is thin if $\alpha$ factors through a proper closed subset of $X$. An arc which is not thin is called a fat arc. An irreducible constructible subset $C$ in $X_{\infty}$ is called a thin set if the generic point of $C$ is thin. An irreducible constructible subset in $X_{\infty}$ which is not thin is called a fat set.

Definition 2.3. $\quad$ Let $\alpha: \operatorname{Spec} K[[t]] \rightarrow X$ be a fat arc of a variety $X$ and $\alpha^{*}: \mathcal{O}_{X, \alpha(0)} \rightarrow K[[t]]$ be the local homomorphism induced from $\alpha$. Suppose that the induced morphism $\operatorname{Spec} K \rightarrow X$ is not dominant. By Proposition 2.5, (i) in [I2], $\alpha^{*}$ is extended to the injective homomorphism of fields $\alpha^{*}: \mathbb{C}(X) \rightarrow K((t))$, where $\mathbb{C}(X)$ is the rational function field of $X$. Define a $\operatorname{map} \operatorname{val}_{\alpha}: \mathbb{C}(X) \backslash\{0\} \rightarrow \mathbb{Z}$ by

$$
\operatorname{val}_{\alpha}(f)=\operatorname{ord}_{t}\left(\alpha^{*}(f)\right)
$$

The function $\operatorname{val}_{\alpha}$ is a discrete valuation of $\mathbb{C}(X)$. We call it the valuation corresponding to $\alpha$. If $\alpha$ is the generic point of an (irreducible and fat) constructible set $C$, the valuation $\operatorname{val}_{\alpha}$ is also denoted by val $C$, and is called the


A fat arc $\alpha$ of $X$ is called a divisorial arc if $\operatorname{val}_{\alpha}$ is a divisorial valuation over $X$. A fat set $C$ is called a divisorial set if the valuation $\operatorname{val}_{C}$ is a divisorial valuation over $X$.

Remark 2.4. For every irreducible, fat set $C \subset X_{\infty}$ and every regular function $f$ on $X$, we have $\operatorname{val}_{C}(f)=\min \left\{\operatorname{ord}_{\gamma}(f) \mid \gamma \in C\right\}$.

Definition 2.5. Let $\psi_{m}: X_{\infty} \rightarrow X_{m}$ be the canonical projection to the space of $m$-jets $X_{m}$. A subset $C \subset X_{\infty}$ is called a cylinder if there is a constructible set $\Sigma \subset X_{m}$ for some $m \in \mathbb{N}$ such that

$$
C=\psi_{m}^{-1}(\Sigma)
$$

Definition 2.6 ([ELM]). For an ideal sheaf $\mathfrak{a}$ on a variety $X$, we define

$$
\operatorname{Cont}^{m}(\mathfrak{a})=\left\{\alpha \in X_{\infty} \mid \operatorname{ord}_{\alpha}(\mathfrak{a})=m\right\}
$$

and

$$
\operatorname{Cont}^{\geq m}(\mathfrak{a})=\left\{\alpha \in X_{\infty} \mid \operatorname{ord}_{\alpha}(\mathfrak{a}) \geq m\right\}
$$


These subsets are called contact loci of the ideal $\mathfrak{a}$. The subset $\operatorname{Cont}^{\geq m}(\mathfrak{a})$ is closed and $\operatorname{Cont}^{m}(\mathfrak{a})$ is locally closed; both are cylinders. If $Z=Z(\mathfrak{a})$, then $\operatorname{Cont}^{m}(\mathfrak{a})$ and $\operatorname{Cont}^{\geq m}(\mathfrak{a})$ are sometimes denoted by $\operatorname{Cont}^{m}(Z)$ and Cont $^{\geq m}(Z)$, respectively.

Definition 2.7 ([ELM]). For a simple normal crossing divisor $E=$ $\bigcup_{i=1}^{s} E_{i}$ on a non-singular variety $X$, we introduce the multi-contact locus for a multi-index $\nu=\left(\nu_{1}, \ldots \nu_{s}\right) \in \mathbb{Z}_{\geq 0}^{s}$ :

$$
\operatorname{Cont}^{\nu}(E)=\left\{\alpha \in X_{\infty} \mid \operatorname{ord}_{\alpha}\left(I_{E_{i}}\right)=\nu_{i} \text { for every } i\right\},
$$

where $I_{E_{i}}$ is the defining ideal of $E_{i}$. If all intersections among the $E_{i}$ are irreducible, then each of these multi-contact loci $\operatorname{Cont}^{\nu}(E)$ is irreducible whenever it is not empty.

Definition 2.8. Let $f: Y \rightarrow X$ be a resolution of the singularities of $X$, and suppose that $E$ is an irreducible smooth divisor on $Y$. For any $q \in \mathbb{Z}_{+}$, we define

$$
W(E, q)=\overline{f_{\infty}\left(\operatorname{Cont}^{q}(E)\right)}
$$

and call it a maximal divisorial set. For $v=q \operatorname{val}_{E}$ we denote sometimes $W(E, q)$ by $W(v)$. When we should clarify the space $X$ with $W(E, q)=W(v) \subset$ $X_{\infty}$ we denote $W(E, q)$ by $W_{X}(E, q)$ or $W_{X}(v)$.

Remark 2.9. It follows by [I3, Proposition 3.4] that the above definition of maximal divisorial set agrees with the one given in [I3, Definition 2.8].

Remark 2.10. The set $W(E, q)$ only depends on the valuation $v=q \mathrm{val}_{E}$, and not on the particular model $Y$ we have chosen; this justify the notation $W(v)$. Moreover, let $g: X^{\prime} \rightarrow X$ be a proper birational morphism of normal varieties, and let $U \subset X^{\prime}$ be an open subset intersecting the center of $v$ on $X^{\prime}$. We consider $v$ also as a divisorial valuation over $X^{\prime}$ and over $U$. Since we can assume that $v=q \mathrm{val}_{E}$ for some smooth divisor $E$ on a resolution of $X^{\prime}$, it follows immediately from our definition of maximal divisorial set that $W_{X}(v)=\overline{g_{\infty}\left(W_{X^{\prime}}(v)\right)}=\overline{g_{\infty}\left(W_{U}(v)\right)}$ (cf. [I3, Proposition 2.9]).

Remark 2.11. The set $W(E, q)$ is a divisorial set corresponding to the valuation $q \mathrm{val}_{E}$ and has the following "maximality" property: any divisorial set $C$ with $\operatorname{val}_{C}=q \operatorname{val}_{E}$ is contained in $W(E, q)$ (see [I3]).

The following is a generalization of a result of [ELM]. 
Proposition 2.12. Let $X=\operatorname{Spec} A$ be an affine variety, and let $\mathfrak{a} \subset A$ be a non-zero ideal. Then, for any $m \in \mathbb{N}$, every fat irreducible component of Cont $^{\geq m}(\mathfrak{a})$ is a maximal divisorial set.

Proof. Let $\varphi: Y \rightarrow X$ be a log-resolution of $\mathfrak{a}$, and write $\mathfrak{a} \cdot \mathcal{O}_{Y}=$ $\mathcal{O}_{Y}\left(-\sum_{i=1}^{s} r_{i} E_{i}\right)$, where $E=\bigcup_{i=1}^{s} E_{i}$ is a simple normal crossing divisor on $Y$. By [ELM, Theorem 2.1], we have

$$
\operatorname{Cont}^{\geq m}(\mathfrak{a}) \supset \underset{\sum r_{i} \nu_{i} \geq m}{\varphi_{\infty}}\left(\operatorname{Cont}^{\nu}(E)\right),
$$

where the complement in $\operatorname{Cont}^{\geq m}(\mathfrak{a})$ of the above union is thin.

We claim that there are only finite number of maximal divisorial sets $\overline{\varphi_{\infty}\left(\operatorname{Cont}^{\nu}(E)\right)}$ 's in $X_{\infty}$, for all possible values of $\nu$. This follows by the following two facts:

(i) We have $\nu \leq \nu^{\prime}$ if and only if $\overline{\operatorname{Cont}^{\nu}(E)} \supset \operatorname{Cont}^{\nu^{\prime}}(E)$, where the partial order $\leq$ in $\mathbb{Z}_{\geq 0}^{s}$ is defined by

$$
\left(\nu_{1}, \ldots, \nu_{s}\right) \leq\left(\nu_{1}^{\prime}, \ldots, \nu_{s}^{\prime}\right) \text { if } \nu_{i} \leq \nu_{i}^{\prime} \text { for all } i .
$$

(ii) The number of minimal elements of $\left\{\nu \in \mathbb{Z}_{\geq 0}^{s} \mid \sum r_{i} \nu_{i} \geq m\right\}$ according to this order $\leq$ is finite.

Then the maximal $\left.\overline{\varphi_{\infty}\left(\operatorname{Cont}^{\nu}(E)\right.}\right)$ 's are the fat components of Cont ${ }^{\geq m}(\mathfrak{a})$. By [ELM, Corollary 2.6], $\overline{\operatorname{Cont}^{\nu}(E)}$ 's are divisorial sets. Therefore, a fat irreducible component of $\operatorname{Cont}^{\geq m}(\mathfrak{a})$ is a divisorial set. To show the maximality, let $C$ be a fat component of $\operatorname{Cont}^{\geq m}(\mathfrak{a})$ and $\alpha \in C$ the generic point. Let $\operatorname{val}_{\alpha}=q \mathrm{val}_{F}$. Then, it is clear that $C \subset W(F, q)$ by the maximality of $W(F, q)$. For the opposite inclusion, take the generic point $\beta \in W(F, q)$. Then it follows that $\operatorname{val}_{\beta}=\operatorname{val}_{\alpha}$, which means that $\operatorname{ord}_{\beta}(f)=\operatorname{ord}_{\alpha}(f)$ for every $f \in K(X)$. This gives $\beta \in$ Cont $^{\geq m}(\mathfrak{a})$, and therefore $W(F, q)$ is contained in a fat irreducible component of Cont ${ }^{\geq m}(\mathfrak{a})$. In conclusion, $C=W(F, q)$.

\section{$\S 3 . \quad$ Codimension of a Maximal Divisorial Set}

In this section we give an extension of the formula on the codimension of maximal divisorial sets established in [ELM] to singular varieties. Let $X$ be an arbitrary complex variety, and let $n=\operatorname{dim} X$. Let $\mathcal{J}_{X} \subset \mathcal{O}_{X}$ be the Jacobian ideal sheaf of $X$. In a local affine chart this ideal is defined as follows. Restrict 
$X$ to an affine chart, and embed it in some $\mathbb{A}^{d}$, so that it is defined by a set of equations

$$
f_{1}\left(u_{1}, \ldots, u_{d}\right)=\cdots=f_{r}\left(u_{1}, \ldots, u_{d}\right)=0 .
$$

Then $\partial_{X}$ is locally defined, in this chart, by the $d-n$ minors of the Jacobian matrix $\left(\partial f_{j} / \partial u_{i}\right)$. Let $S \subset X$ be subscheme defined by $\mathcal{J}_{X}$. Note that $S$ is supported precisely over the singular locus of $X$.

We decompose

$$
X_{\infty} \backslash S_{\infty}=\bigsqcup_{e=0}^{\infty} X_{\infty}^{e}, \quad \text { where } X_{\infty}^{e}:=\left\{\gamma \in X_{\infty} \mid \operatorname{ord}_{\gamma}\left(\mathcal{J}_{X}\right)=e\right\}
$$

and let $X_{m, \infty}:=\psi_{m}\left(X_{\infty}\right)$ and $X_{m, \infty}^{e}:=\psi_{m}\left(X_{\infty}^{e}\right)$, where $\psi_{m}: X_{\infty} \rightarrow X_{m}$ is the truncation map. Also, let

$$
X_{\infty}^{\leq e}:=\left\{\gamma \in X_{\infty} \mid \operatorname{ord}_{\gamma}\left(\mathcal{J}_{X}\right) \leq e\right\} \quad \text { and } \quad X_{m, \infty}^{\leq e}:=\psi_{m}\left(X_{\infty}^{\leq e}\right) .
$$

We will need the following geometric lemma on the fibers of the truncation maps. A weaker version of this property was proven by Denef and Loeser in [DL, Lemma 4.1]; the sharper stated here is taken from [EM2, Proposition 4.1].

Lemma 3.1 ([DL], [EM2]). For $m \geq e$, the morphism $X_{m+1, \infty}^{e} \rightarrow$ $X_{m, \infty}^{e}$ is a piecewise trivial fibration with fibers isomorphic to $\mathbb{A}^{n}$.

Definition 3.2. $\quad$ Let $C$ and $C^{\prime}$ be two constructible sets in $X_{\infty}$ that are not contained in $S_{\infty}$. We denote $C \sim C^{\prime}$ if $\bar{C} \cap\left(X_{\infty} \backslash S_{\infty}\right)=\overline{C^{\prime}} \cap\left(X_{\infty} \backslash S_{\infty}\right)$. A constructible set $C$ in $X_{\infty}$ that is not contained in $S_{\infty}$ is called a quasi-cylinder if there is a cylinder $C^{\prime}$ such that $C \sim C^{\prime}$.

Remark 3.3. The relation $\sim$ is clearly an equivalence relation. Note also that the closure of a cylinder which is not contained in $S_{\infty}$ is a quasi-cylinder.

Let $C$ be an irreducible quasi-cylinder. By Lemma 3.1, we can also define the codimension of $C$ in $X_{\infty}$. Indeed, for a cylinder $C^{\prime}$ such that $C^{\prime} \sim C$, one can check that the codimension of

$$
C^{\prime} \underset{m}{m}:=\psi_{m}\left(C^{\prime}\right) \cap X_{m}^{\leq e}, \infty
$$

inside $X_{m, \infty}^{\leq e}$ stabilizes for $m \gg e$ (this is done in detail in Section 5 of [EM2]), and thus we can define

$$
\operatorname{codim}\left(C^{\prime}, X_{\infty}\right):=\operatorname{codim}\left(C^{\prime} \underset{m}{\leq e}, X_{m}^{\leq e}, \infty\right) \quad \text { for } m \gg e .
$$


Here, we note that the condition $C^{\prime} \underset{m}{\leq e}$ is not empty is equivalent to the condition $e \geq \operatorname{ord}_{\alpha}\left(\mathcal{J}_{X}\right)$, where $\alpha \in C^{\prime}$ is the generic point, and therefore $C_{m}^{\prime \leq e}$ is indeed nonempty for $e \gg 0$, since $C^{\prime} \nsubseteq S_{\infty}$. Observe that $C_{m}^{\prime \leq e}$ is open, hence dense, in $\psi_{m}\left(C^{\prime}\right)$. Note also that $\operatorname{codim}\left(C^{\prime}, X_{\infty}\right)=\operatorname{codim}\left(\overline{C^{\prime}}, X_{\infty}\right)$, since $\psi_{m}\left(C^{\prime}\right) \subseteq$ $\psi_{m}\left(\overline{C^{\prime}}\right) \subseteq \overline{\psi_{m}\left(C^{\prime}\right)}$. Then we define

$$
\operatorname{codim}\left(C, X_{\infty}\right):=\operatorname{codim}\left(C^{\prime}, X_{\infty}\right)
$$

Notice that this definition does not depend on the choice of $C^{\prime}$. Indeed if $C^{\prime \prime}$ is another cylinder with $C^{\prime \prime} \sim C$, then we have $C^{\prime} \sim C^{\prime \prime}$. This implies that the symmetric difference between $\overline{C^{\prime}}$ and $\overline{C^{\prime \prime}}$ is contained in $S_{\infty}$. We deduce that $\operatorname{codim}\left(\overline{C^{\prime \prime}}, X_{\infty}\right)=\operatorname{codim}\left(\overline{C^{\prime}}, X_{\infty}\right)$ and hence $\operatorname{codim}\left(C^{\prime \prime}, X_{\infty}\right)=$ $\operatorname{codim}\left(C^{\prime}, X_{\infty}\right)$.

Remark 3.4. If $C$ is an irreducible cylinder, then this definition of codimension coincides with the "usual" definition of codimension, i.e., the maximal length $r$ of a sequence $C=C_{0} \subsetneq C_{1} \subsetneq \cdots \subsetneq C_{r}=X_{\infty}$ of irreducible closed subsets of $X_{\infty}$. Indeed, if $s$ the codimension of $C$ as defined above, then the inequality

$$
r \geq s
$$

is obvious by definition of $s$. For the opposite inequality we note that $s=$ $\operatorname{codim}\left(\psi_{m}(C), X_{m, \infty}\right)$ for $m \gg 0$. Then the opposite inequality is obtained as follows: from the sequence,

$$
C=C_{0} \subsetneq C_{1} \subsetneq \cdots \subsetneq C_{r}
$$

of irreducible closed subsets of $X_{\infty}$, we have the sequence

$$
\overline{\psi_{m}(C)}=\overline{\psi_{m}\left(C_{0}\right)} \subsetneq \overline{\psi_{m}\left(C_{1}\right)} \subsetneq \cdots \subsetneq \overline{\psi_{m}\left(C_{r}\right)}
$$

for $m \gg 0$, since $C_{i}=\lim _{\longleftarrow} \overline{\psi_{m}\left(C_{i}\right)}$. This yields $r \leq s$.

For a non-singular variety $X$, every component of a cylinder is fat ([ELM]), but this is no longer true in the singular case, as the following example show.

Example 3.5. Let $X$ be the Whitney Umbrella, i.e. a hypersurface in $\mathbb{C}^{3}$ defined by $x y^{2}-z^{2}=0$. For $m \geq 1$, let $\alpha_{m}: \mathbb{C}[x, y, z] /\left(x y^{2}-z^{2}\right) \rightarrow$ $\mathbb{C}[t] /\left(t^{m+1}\right)$ be the $m$-jet defined by $\alpha_{m}(x)=t, \alpha_{m}(y)=0, \alpha_{m}(z)=0$. Then, the cylinder $\psi_{m}^{-1}\left(\alpha_{m}\right)$ is contained in $\operatorname{Sing}(X)_{\infty}$, where $\operatorname{Sing}(X)=(y=z=0)$.

Proposition 3.6. Let $X$ be a reduced scheme. The number of irreducible components of a cylinder on $X_{\infty}$ is finite. 
Proof. First, we show the number of irreducible components of a cylinder that are not contained in $S_{\infty}$ is finite. Let $C=\psi_{m}^{-1}(\Sigma)$ be a cylinder over a constructible set $\Sigma \subset X_{m}$. Let $\varphi: Y \rightarrow X$ be a resolution of the singularities of $X$, and assume that $\varphi$ is an isomorphism away from $S$. As $\varphi$ is isomorphic away from $S$, by the valuative criteria for the properness, the generic point of each component of $C$ not contained in $S_{\infty}$ can be lifted to the generic point of a component of the cylinder $\varphi_{\infty}^{-1}(C)=\left(\psi_{m}^{Y}\right)^{-1} \varphi_{m}^{-1}(\Sigma)$. The finiteness of the components of $C$ not contained in $S_{\infty}$ follows from the finiteness of the components of $\left(\psi_{m}^{Y}\right)^{-1} \varphi_{m}^{-1}(\Sigma)$, as $Y$ is non-singular.

Now, to prove the proposition, we use induction of the dimension. If $\operatorname{dim} X=0$, then the assertion is trivial, since $X_{\infty} \simeq X$ is a finite points set. If $\operatorname{dim} X=n \geq 1$ and assume that the assertion is true for a reduced scheme of dimension $\leq n-1$. Let $F_{1}, \ldots, F_{r}$ be the irreducible components of $C$ not contained in $S_{\infty}$. Let

$$
C^{\prime}:=\overline{\psi_{m}\left(C \backslash \bigcup_{i} F_{i}\right)}
$$

be the closure in $\Sigma \cap S_{m}$. Then, every irreducible component $F$ of $C$ other than $F_{i}$ 's is contained in $\left(\psi_{m}^{S}\right)^{-1}\left(C^{\prime}\right)$. As $F$ is an irreducible component of $C=\psi_{m}^{-1}(\Sigma)$ which contains $\left(\psi_{m}^{S}\right)^{-1}\left(C^{\prime}\right), F$ is also an irreducible component of the cylinder $\left(\psi_{m}^{S}\right)^{-1}\left(C^{\prime}\right)$ of a lower dimensional variety $S$. By the induction hypothesis, we obtain the assertion of our proposition.

The second part of the following corollary also appears as [EM2, Lemma 5.1].

Corollary 3.7. Every fat irreducible component of a cylinder is a quasicylinder, and every thin component of a cylinder is contained in $S_{\infty}$.

Proof. Let $C_{1}, \ldots C_{r}$ be the irreducible components of a cylinder $C$. As $C_{i}=\lim _{\longleftarrow_{m}} \overline{\psi_{m}\left(C_{i}\right)}$, for $m \gg 0$ it follows that

$$
\psi_{m}\left(C_{i}\right) \not \subset \overline{\psi_{m}\left(C_{j}\right)} \quad \text { for } i \neq j \text {. }
$$

Then the non-empty open subset $C_{i} \backslash\left(\bigcup_{j \neq i} \psi_{m}^{-1}\left(\overline{\psi_{m}\left(C_{j}\right)}\right)\right)$ of $C_{i}$ is a cylinder, therefore if $C_{i}$ is fat, then $C_{i}$ is an irreducible quasi-cylinder.

For the second assertion, assume $C_{1}$ is thin. If $C_{1}$ is not contained in $S_{\infty}$, there is $e>0$ such that $X_{\infty}^{\leq e} \cap C_{1} \neq \emptyset$ which is open in $C_{1}$. Let

$$
U:=X_{\infty}^{\leq e} \backslash\left(\bigcup_{j \neq 1} \psi_{m}^{-1}\left(\overline{\psi_{m}\left(C_{j}\right)}\right)\right) \quad \text { and } \quad U_{m}:=X_{m, \infty}^{\leq e} \backslash\left(\bigcup_{j \neq 1} \overline{\psi_{m}\left(C_{j}\right)}\right) .
$$


Then

$$
U \cap C_{1}=\psi_{m}^{-1}\left(U_{m} \cap \psi_{m}\left(C_{1}\right)\right)
$$

is a non-empty open subset of $C_{1}$ for every $m \gg 0$. By Lemma 3.1, the codimension of $U_{m} \cap \psi_{m}\left(C_{1}\right)$ in $X_{m, \infty}$ is bounded. But it is in contradiction with the fact that $C_{1}$ is thin by [M1, Lemma 3.7].

The truncation morphisms induce morphisms

$$
f_{m}: Y_{m} \longrightarrow X_{m, \infty} \subseteq X_{m}
$$

Indeed, the inclusion $f_{m}\left(Y_{m}\right) \subseteq X_{m, \infty}$ is implied by the fact that $Y_{m, \infty}=Y_{m}$, which follows by the smoothness of $Y$. An important ingredient of the proof for our codimension formula, as well as the key ingredient for the change-ofvariable formula in motivic integration, is the following geometric statement on the fibers of these morphisms, due to Denef and Loeser [DL, Lemma 3.4]. For a more precise statement of the following lemma, we refer to [EM2, Theorem 6.2 and Lemma 6.3].

Lemma 3.8 ([DL]). Let $\gamma \in Y_{\infty}$ be any arc such that $\tau:=$ $\operatorname{ord}_{\gamma}\left(\widehat{K}_{Y / X}\right)<\infty$. Then for any $m \geq 2 \tau$, letting $\gamma_{m}=\psi_{m}^{Y}(\gamma)$, we have

$$
f_{m}^{-1}\left(f_{m}\left(\gamma_{m}\right)\right) \cong \mathbb{A}^{\tau}
$$

Moreover, for every $\gamma_{m}^{\prime} \in f_{m}^{-1}\left(f_{m}\left(\gamma_{m}\right)\right)$ we have $\pi_{m, m-\tau}^{Y}\left(\gamma_{m}\right)=\pi_{m, m-\tau}^{Y}\left(\gamma_{m}^{\prime}\right)$, where $\pi_{m, m-\tau}^{Y}: Y_{m} \rightarrow Y_{m-\tau}$ is the canonical truncation morphism.

We obtain the following results.

Theorem 3.9. Let $f: Y \rightarrow X$ be a resolution of the singularities such that $E$ appears as a smooth divisor on $Y$. Then, $W(E, q)$ is a quasi-cylinder of $X_{\infty}$ of codimension

$$
\operatorname{codim}\left(W(E, q), X_{\infty}\right)=q \cdot\left(\operatorname{ord}_{E}\left(\widehat{K}_{Y / X}\right)+1\right),
$$

Proof. Let

$$
\operatorname{Cont}^{q}(E)^{0}:=\left\{\gamma \in Y_{\infty} \mid \operatorname{ord}_{\gamma}(E)=q, \operatorname{ord}_{\gamma}(\overline{\operatorname{Ex}(f) \backslash E})=0\right\} .
$$

This is an open subset of $\operatorname{Cont}^{q}(E)$. Note that $\tau:=\operatorname{ord}_{\gamma}\left(\widehat{K}_{Y / X}\right)$ is the same for all $\gamma \in \operatorname{Cont}^{q}(E)^{0}$. Then, by Lemma 3.8, one can see that

$$
f_{m}^{-1}\left(f_{m}\left(\psi_{m}^{Y}\left(\operatorname{Cont}^{q}(E)^{0}\right)\right)\right)=\psi_{m}^{Y}\left(\operatorname{Cont}^{q}(E)^{0}\right) \quad \text { for all } m \gg 0 .
$$


The fact that $\overline{f_{\infty}\left(\text { Cont }^{q}(E)^{0}\right)}$ is a quasi-cylinder in $X_{\infty}$ follows by the equalities $f_{m} \circ \psi_{m}^{Y}=\psi_{m} \circ f_{\infty}$ and the fact that $\operatorname{Cont}^{q}(E)^{0}$ is a cylinder in $Y_{\infty}$.

Let $\operatorname{ord}_{E} \widehat{K}_{Y / X}=k$ and $e=q \cdot k$. Then $\psi_{m}^{Y}\left(\operatorname{Cont}^{q}(E)\right) \subset$ $\psi_{m}^{Y}\left(\operatorname{Cont}^{e}\left(\widehat{K}_{Y / X}\right)\right)$. By Lemma 3.8, the morphism $\psi_{m}^{Y}\left(\operatorname{Cont}^{e}\left(\widehat{K}_{Y / X}\right)\right) \rightarrow$ $f_{m}\left(\psi_{m}^{Y}\left(\operatorname{Cont}^{e}\left(\widehat{K}_{Y / X}\right)\right)\right)$ induces a morphism

$$
\psi_{m}^{Y}\left(\operatorname{Cont}^{q}(E)\right) \rightarrow f_{m}\left(\psi_{m}^{Y}\left(\operatorname{Cont}^{q}(E)\right)\right)
$$

with irreducible $e$-dimensional fibers for $m \gg e$. Note that $\psi_{m}^{Y}\left(\operatorname{Cont}^{q}(E)\right)$ is an irreducible closed subset of codimension $q$ in $Y_{m}$. Then

$$
\begin{aligned}
\operatorname{dim}\left(f_{m}\left(\psi_{m}^{Y}\left(\operatorname{Cont}^{q}(E)\right)\right)\right) & =\operatorname{dim}\left(\psi_{m}^{Y}\left(\operatorname{Cont}^{q}(E)\right)\right)-e \\
& =m(n+1)-q-e=m(n+1)-q(k+1) .
\end{aligned}
$$

The formula on codimension follows.

Proposition 3.10. The valuation corresponding to a fat irreducible component of a cylinder is the valuation corresponding to an irreducible component of a contact locus.

Proof. Let $C \subset X_{\infty}$ be any fat irreducible component of a cylinder. For every $k \in \mathbb{N}$, define

$$
\mathfrak{a}_{k !}:=\left\{f \in \mathcal{O}_{X} \mid \operatorname{val}_{C}(f) \geq k !\right\} \quad \text { and } \quad B_{k !}:=\left\{\gamma \in X_{\infty} \mid \operatorname{ord}_{\gamma}\left(\mathfrak{a}_{k !}\right) \geq k !\right\} .
$$

Note that we have a chain of inclusions

$$
B_{n !} \supseteq B_{(n+1) !} \supseteq \cdots \supseteq C .
$$

For each $k$, let $c_{k}$ be the smallest codimension in $X_{\infty}$ of irreducible components of $B_{k}$ ! containing $C$, and let $n_{k}$ be the number of such components. Since

$$
\begin{gathered}
c_{k} \leq c_{k+1} \leq \operatorname{codim}\left(C, X_{\infty}\right)<\infty \quad \text { and } \\
\text { if } c_{k}=c_{k+1}, \text { then } n_{k} \geq n_{k+1}>0,
\end{gathered}
$$

the sequences $\left\{c_{k}\right\}$ and $\left\{n_{k}\right\}$ stabilize. Therefore we find a closed subset $W \subset$

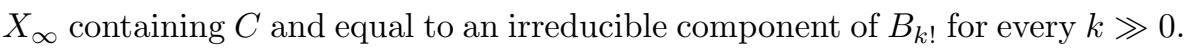
We clearly have $\mathrm{val}_{C} \geq \mathrm{val}_{W}$ on regular functions because of the inclusion $C \subseteq W$. Conversely, let $h \in \mathcal{O}_{X}$ be an arbitrary nontrivial element. We can arrange to have $\operatorname{val}_{C}\left(h^{m}\right)=k$ ! for some $m, k \in \mathbb{N}$. This means that $h^{m} \in \mathfrak{a}_{k !}$, hence we have $\operatorname{val}_{W}\left(h^{m}\right) \geq k$ ! by the definition of $W$. Then we conclude that

$$
\operatorname{val}_{W}(h)=\frac{\operatorname{val}_{W}\left(h^{m}\right)}{m} \geq \frac{\operatorname{val}_{C}\left(h^{m}\right)}{m}=\operatorname{val}_{C}(h) .
$$




\section{$\S 4$. Determination of a Divisorial Valuation by Finite Data}

Let $X=\operatorname{Spec} A$ be an affine variety, and let $v$ be a divisorial valuation over $X$, i.e., $v=q \operatorname{val}_{E}$ for $q \in \mathbb{N}$ and a divisor $E$ over $X$. For a subset $V \subset X_{\infty}$ we denote the set of fat $\operatorname{arcs}$ in $V$ by $V^{o}$.

Lemma 4.1. With the above notation, let $x_{1}, \ldots, x_{m}$ be elements in $A$, and denote by

$$
\varphi: Y=\operatorname{Spec} A\left[\frac{x_{2}}{x_{1}}, \ldots, \frac{x_{m}}{x_{1}}\right] \rightarrow X=\operatorname{Spec} A
$$

the canonical birational morphism. If

$$
\Sigma=\overline{\bigcap_{j=1}^{r}\left(\operatorname{Cont}^{v_{j}}\left(f_{j}\right)\right)^{o}}
$$

is an irreducible subset in $Y_{\infty}$ for some $f_{1}, \ldots, f_{r} \in A\left[\frac{x_{2}}{x_{1}}, \ldots, \frac{x_{m}}{x_{1}}\right]$ and $v_{1}, \ldots, v_{r} \in \mathbb{N}$, then

$$
\overline{\varphi_{\infty}(\Sigma)}=\overline{\left(\bigcap_{j=1}^{r} \operatorname{Cont}_{j}^{v_{j}^{\prime}}\left(f_{j}^{\prime}\right)\right) \cap\left(\bigcap_{i=1}^{m} \operatorname{Cont}^{p_{i}}\left(x_{i}\right)\right)^{o}}
$$

for some $v_{j}^{\prime}, p_{i} \in \mathbb{N}$ and $f_{j}^{\prime} \in A$.

Proof. First we define $v_{j}^{\prime}, p_{i}$ and $f_{j}^{\prime}(i=1, \ldots, m, j=1, \ldots, r)$. Let $\tilde{\alpha}$ be the generic point of $\Sigma$ and $\alpha=\varphi_{\infty}(\tilde{\alpha})$. Let $p_{i}=\operatorname{ord}_{\alpha}\left(x_{i}\right) \geq 0$. As $\alpha$ has the lifting $\tilde{\alpha}$ on $Y$, we have $\operatorname{ord}_{\alpha}\left(x_{i}\right)-\operatorname{ord}_{\alpha}\left(x_{1}\right)=\operatorname{ord}_{\tilde{\alpha}}\left(x_{i} / x_{1}\right) \geq 0$, which means $p_{1} \leq p_{i}$ for every $i=2, \ldots, m$.

Now for $f_{j} \in A\left[\frac{x_{2}}{x_{1}}, \ldots, \frac{x_{m}}{x_{1}}\right]$, let the minimal $a$ such that $f_{j} x_{1}^{a} \in A$ be $a_{j}$ and let $f_{j}^{\prime}=f_{j} x_{1}^{a_{j}}$. Next let $v_{j}^{\prime}=a_{j} p_{1}+v_{j}$. Then it is clear that

$$
\alpha \in\left(\bigcap_{j=1}^{r} \operatorname{Cont}^{v_{j}^{\prime}}\left(f_{j}^{\prime}\right)\right) \cap\left(\bigcap_{i=1}^{m} \operatorname{Cont}^{p_{i}}\left(x_{i}\right)\right)^{o} .
$$

Therefore, the inclusion of the left side of (4) in the right side follows. For the converse inclusion, take any arc $\beta \in\left(\bigcap_{j=1}^{r} \operatorname{Cont}^{v_{j}^{\prime}}\left(f_{j}^{\prime}\right)\right) \cap\left(\bigcap_{i=1}^{m} \operatorname{Cont}^{p_{i}}\left(x_{i}\right)\right)^{o}$. Then, by the condition $p_{1} \leq p_{i}(i>1), \beta$ has the lifting $\tilde{\beta}$ on Y. Hence, $\operatorname{ord}_{\tilde{\beta}}\left(f_{j}\right)=\operatorname{ord}_{\beta}\left(f_{j}^{\prime}\right)-\operatorname{ord}_{\beta}\left(x_{1}^{a_{j}}\right)=v_{j}$ which implies that $\tilde{\beta} \in \Sigma$. 
Theorem 4.2. Let $X=\operatorname{Spec} A$ be an affine variety. For any divisorial valuation $v$ over $X$, there are finite number of functions $f_{1}, \ldots, f_{r} \in A$ and positive integers $v_{1}, \ldots, v_{r}$ such that

$$
W(v)=\overline{\bigcap_{i=1}^{r}\left(\operatorname{Cont}^{v_{i}}\left(f_{i}\right)\right)^{o}} .
$$

Equivalently, we have $W(v) \sim \bigcap_{i=1}^{r} \operatorname{Cont}^{v_{i}}\left(f_{i}\right)$.

Proof. For a divisor $E$ over $X$ such that $v=q \mathrm{val}_{E}$, there exists a resolution of singularities $\varphi: Y \rightarrow X$ such that $E$ appears as a smooth divisor on $Y$. Then, $\varphi$ is obtained by blowing up an ideal $I$ in $A$. Let $g_{1}, \ldots, g_{m}$ be generators of $I$. Then $Y$ is covered by affine open subsets $U_{i}=\operatorname{Spec} A\left[\frac{g_{1}}{g_{i}}, \ldots, \frac{g_{m}}{g_{i}}\right],(i=1, \ldots, m)$. Take an $i$ such that $U_{i} \cap E \neq \emptyset$. Let this $i$ be 1 . Let $B=A\left[\frac{g_{2}}{g_{1}}, \ldots, \frac{g_{m}}{g_{1}}\right]$ and consider the restricted morphism

$$
\varphi: U_{1}=\operatorname{Spec} B \rightarrow X=\operatorname{Spec} A .
$$

Let the defining ideal of $E$ on $U_{1}$ be $J=\left(h_{1}, \ldots, h_{s}\right)$. Then the blowing-up $\psi: U_{1}^{\prime} \rightarrow U_{1}$ of the ideal $J$ is an isomorphism, because it is the blow up with the center Cartier divisor $E$. Hence $U_{1}^{\prime}$ is non-singular and it is covered by charts $V_{j}=\operatorname{Spec} B\left[\frac{h_{1}}{h_{j}}, \ldots, \frac{h_{s}}{h_{j}}\right](j=1, \ldots, s)$. Take $j$ such that $V_{j} \cap E \neq \emptyset$. Let this $j$ be 1 . Then, on $V_{1}$ the divisor $E$ is a principal divisor defined by $h_{1}$. Therefore, on $V_{1}$, we have $W_{V_{1}}(v)=\overline{\left(\operatorname{Cont}^{q}\left(h_{1}\right)\right)^{o}}$. Applying Lemma 4.1 for

$$
\psi: V_{1}=\operatorname{Spec} B\left[\frac{h_{2}}{h_{1}}, \ldots, \frac{h_{s}}{h_{1}}\right] \rightarrow U_{1}=\operatorname{Spec} B,
$$

and the property in Remark 2.10 , we obtain that

$$
W_{U_{1}}(v)=\overline{\psi_{\infty}\left(W_{V_{1}}(v)\right)}=\overline{\left(\operatorname{Cont}^{q}\left(h_{1}\right) \cap \operatorname{Cont}^{p_{2}}\left(h_{2}\right) . . \cap \operatorname{Cont}^{p_{s}}\left(h_{s}\right)\right)^{o}}
$$

for some $p_{2}, \ldots, p_{s}$. Next, applying the lemma and the remark again to

$$
\varphi: U_{1}=\operatorname{Spec} B \rightarrow X=\operatorname{Spec} A,
$$

we obtain

$$
W_{X}(v)=\overline{\varphi_{\infty}\left(W_{U_{1}}(v)\right)}
$$

$=\overline{\left(\operatorname{Cont}^{q^{\prime}}\left(h_{1}^{\prime}\right) \cap \operatorname{Cont}^{p_{2}^{\prime}}\left(h_{2}^{\prime}\right) \cap \ldots \operatorname{Cont}^{p_{s}^{\prime}}\left(h_{s}^{\prime}\right) \cap \operatorname{Cont}^{q_{1}}\left(g_{1}\right) \cap \ldots \cap \operatorname{Cont}^{q_{m}}\left(g_{m}\right)\right)^{o}}$

for some $h_{j}^{\prime} \in A, q^{\prime}, p_{i}^{\prime}, q_{l} \in \mathbb{N},(1 \leq j \leq s, i \geq 2$, and $1 \leq l \leq m)$. 
Remark 4.3. By this theorem, it follows that $W(v)$ is the unique fat component of the quasi-cylinder $\overline{\bigcap_{i=1}^{r} \operatorname{Cont}^{v_{i}}\left(f_{i}\right)}$. As a thin component of a quasi-cylinder is in $S_{\infty}$ by Corollary 3.7, we see that the inclusion (1) is indeed an equality off $S_{\infty}$, which implies Theorem 0.2. The fact that $W(v)$ is a quasicylinder, which was proven in Theorem 3.9, also follows immediately from this theorem.

Definition 4.4. Under the situation of Theorem 4.2, we call $v\left(f_{1}\right)=$ $q_{1}, \ldots, v\left(f_{r}\right)=q_{r}$ the generating conditions, or simply the generators, of $v$.

Actually, the conditions in the above definition "generate" or "determine" the divisorial valuation $v$ in the following sense.

Corollary 4.5. Let $v$ be a divisorial valuation over $X=\operatorname{Spec} A$. Then there exist $f_{1}, \ldots, f_{r} \in A$ and $v_{1}, \ldots, v_{r} \in \mathbb{N}$ such that for every $f \in A \backslash\{0\}$ $v(f)=\min \left\{v^{\prime}(f) \mid v^{\prime}\right.$ is a divisorial valuation over $X$ such that $\left.v^{\prime}\left(f_{i}\right)=v_{i}\right\}$.

Proof. Let $v\left(f_{i}\right)=v_{i}(i=1, \ldots, r)$ be generators of $v$. For every $f \in$ $A \backslash\{0\}$, let $v_{0}(f)$ be

$\min \left\{v^{\prime}(f) \mid v^{\prime}\right.$ is a divisorial valuation over $X$ such that $\left.v^{\prime}\left(f_{i}\right)=v_{i}\right\}$.

Then it is clear that $v_{0}(f) \leq v(f)$ for every $f \in A \backslash\{0\}$ by the definition of $v_{0}$. On the other hand for an arbitrary $f \in A \backslash\{0\}$, take a divisorial valuation $v^{\prime}$ over $X$ (depending on $f$ ) such that $v^{\prime}(f)=v_{0}(f)$ and $v^{\prime}\left(f_{i}\right)=v_{i}(i=1, \ldots, r)$. Let $\beta$ be the generic point of $W\left(v^{\prime}\right)$. Then $\beta \in \overline{\bigcap_{i=1}^{r}\left(\operatorname{Cont}^{v_{i}}\left(f_{i}\right)\right)^{o}}=W(v)$. The inclusion $W\left(v^{\prime}\right) \subset W(v)$ yields the inequality $\left.v^{\prime}\right|_{A} \geq\left. v\right|_{A}$. Therefore, in particular, we have $v_{0}(f)=v^{\prime}(f) \geq v(f)$.

Remark 4.6. Suppose that $K$ is the function field of an $n$-dimensional variety and $x$ is an element purely transcendental over $K$. Suppose that $v$ is a divisorial valuation for $K(x)$ with residue field L. Let $w$ be the restriction of $v$ to $K$. Denote by $L^{\prime}$ the residue field of $w$. MacLane's theorem says that one can construct $v$ from $w$ by knowing the values of $v$ on a sequence of key polynomials in $K[x]$ (see [ML]). If the sequence is infinite, then $L$ is algebraic over $L^{\prime}$. Then the transcendence degree of $L$ over $\mathbb{C}$ would be at most $n-1$ and $v$ can not be divisorial. Hence $v$ can be determined from $w$ and the values of $v$ on a finite number of key polynomials. Inductively, we see that we can determine a divisorial valuation $v$ on a finitely generated purely transcendental field over $\mathbb{C}$ by knowing the values of $v$ on just finitely many elements in the function fields. 
Example 4.7 (Divisorial valuations over $\mathbb{C}^{2}$ ). We give some explicit construct of divisorial valuations of $\mathbb{C}(x, y)$ in terms of blow-ups of $\mathbb{C}^{2}$. We will denote by $v_{1}, . ., v_{4}, v_{5}$ and $v_{5}^{\prime}$ the valuations $\operatorname{val}_{E_{1}}, . ., \operatorname{val}_{E_{4}}, \mathrm{val}_{E_{5}}$ and $\operatorname{val}_{E_{5}^{\prime}}$ associated to the following exceptional divisors $E_{1}, . ., E_{4}, E_{5}$ and $E_{5}^{\prime}$.

(i) Let $E_{1}$ be the exceptional divisor of the blowing-up $\varphi_{1}: X_{1} \rightarrow \mathbb{C}^{2}$ with the center $(x, y)=(0,0)$. Then, the generators of $v_{1}$ are $v_{1}(x)=v_{1}(y)=1$ and $v_{1}$ is a toric valuation.

(ii) Let $E_{2}$ be the exceptional divisor of the blowing-up $\varphi_{2}: X_{2} \rightarrow X_{1}$ with the center $\left(x_{1}, y_{1}\right)=(0,0)$, where $x_{1}=x, y_{1}=y / x$. Then, the generators of $v_{2}$ are $v_{2}(x)=1, v_{2}(y)=2$ and $v_{2}$ is a toric valuation.

(iii) Let $E_{3}$ be the exceptional divisor of the blowing-up $\varphi_{3}: X_{3} \rightarrow X_{2}$ with the center $\left(x_{2}, y_{2}\right)=(0,0)$, where $x_{2}=x_{1} / y_{1}, y_{2}=y_{1}$. Then, the generators of $v_{3}$ are $v_{3}(x)=2, v_{3}(y)=3$ and $v_{3}$ is a toric valuation.

(iv) Let $E_{4}$ be the exceptional divisor of the blowing-up $\varphi_{4}: X_{4} \rightarrow X_{3}$ with the center $\left(x_{3}, y_{3}\right)=(0, \lambda)(\lambda \in \mathbb{C} \backslash\{0\})$, where $x_{3}=x_{2}, y_{3}=y_{2} / x_{2}$. Then, the generators of $v_{4}$ are $v_{4}(x)=2, v_{4}(y)=3$ and $v_{4}\left(y^{2}-\lambda x^{3}\right)=7$.

(v) Let $E_{5}$ be the exceptional divisor of the blowing-up $\varphi_{5}: X_{5} \rightarrow X_{4}$ with the center $\left(x_{4}, y_{4}\right)=(0,0)$, where $x_{4}=x_{3}, y_{4}=\left(y_{3}-\lambda\right) / x_{3}$. Then, the generators of $v_{5}$ are $v_{5}(x)=2, v_{5}(y)=3$ and $v_{5}\left(y^{2}-\lambda x^{3}\right)=8$.

(v') Let $E_{5}^{\prime}$ be the exceptional divisor of the blowing-up $\varphi_{5}^{\prime}: X_{5}^{\prime} \rightarrow X_{4}$ with the center $\left(x_{4}, y_{4}\right)=(0, \mu)(\mu \in \mathbb{C} \backslash\{0\})$. Then, the generators of $v_{5}^{\prime}$ are $v_{5}^{\prime}(x)=2, v_{5}^{\prime}(y)=3, v_{5}^{\prime}\left(y^{2}-\lambda x^{3}\right)=7$ and $v_{5}^{\prime}\left(y^{3}-\lambda x^{3} y-\mu x^{5}\right)=11$.

Example 4.8 (Divisorial valuations over $\mathbb{C}^{3}$ ). In $[\mathrm{CGP}]$ V. Cossart, C. Galindo and O. Piltant showed an example of divisorial valuation $v$ with the center at a non singular point $x \in X$ of a three dimensional variety such that the graded ring $\operatorname{Gr}_{v}\left(\mathcal{O}_{X, x}\right)$ associated to this valuation is not Noetherian. But this valuation is also "finitely generated" in our sense.

In [CGP] the valuation $v$ is obtained as follows: Let $R$ be a regular local ring of dimension three and let $X_{0}=\operatorname{Spec} R$. Let $X_{1} \rightarrow X_{0}$ be the blow up at the closed point of $X_{0}, E_{1}=\operatorname{Proj} \mathbb{C}[x, y, z]$ the exceptional divisor and $C$ the cubic in $E_{1}$ defined by the equation $x^{2} z+x y^{2}+y^{3}=0$. Take the blow up $X_{2} \rightarrow X_{1}$ at the smooth point of $C$ with the homogeneous coordinates $(1: 0: 0)$. Then, for $n \geq 10$ construct a sequence $X_{n} \rightarrow X_{n-1} \rightarrow \cdots \rightarrow X_{2}$, where each $X_{i+1} \rightarrow X_{i}$ is the blow up at the intersection of the strict transform of $C$ and the exceptional divisor of $X_{i} \rightarrow X_{i-1}$. For simplicity, we assume that 
$R$ is the local ring at the origin of $X=\operatorname{Spec} \mathbb{C}[x, y, z]$. As $\operatorname{Gr}_{v}(R)$ is not Noetherian, the graded ring $\operatorname{Gr}_{v}(\mathbb{C}[x, y, z])$ is also non-Noetherian. But on $X$ we have finite "generators": $v(x)=n-1, v(y)=n, v(z)=n+1$ and $v\left(x^{2} z+x y^{2}+y^{3}\right)=4(n-1)$.

\section{$\S 5 . \quad$ Characterization of Toric Valuations}

Using the result of [ELM], Theorem 0.3 follows immediately. Although it is a particular case of the general statement for toric varieties, it is instructive to give the proof independently.

Proof of Theorem 0.3. Let $X=\mathbb{C}^{n}$, and consider the set

$$
C:=\left\{\gamma \in X_{\infty} \mid \operatorname{ord}_{\gamma}\left(x_{i}\right) \geq a_{i}\right\} .
$$

It is immediate to check that this is an irreducible closed cylinder of codimension $\sum a_{i}$ in $X_{\infty}$, and in fact it coincides with the maximal divisorial set associated with the toric valuation given by assigning weights $a_{i}$ to the coordinates $x_{i}$. Thus the statement follows as long as we show that $W(v) \subset X_{\infty}$ is equal to $C$. By the first condition in (2), we have $W(v) \subseteq C$, whereas the second condition and Theorem 3.9 imply that $\operatorname{codim}\left(W, X_{\infty}\right) \leq \operatorname{codim}\left(C, X_{\infty}\right)$. We conclude that $W(v)=C$ by the irreducibility of $C$.

Using notation as in [F], let $M$ be a free abelian group of rank $n \geq 1$, and let $N=M^{*}$ be its dual. Fix a maximal dimensional rational polyhedral cone $\sigma \subset N \otimes \mathbb{R}$, and let $\Delta$ be the fan consisting of all faces of $\sigma$. Denote $R_{\sigma}=\sigma^{\vee} \cap M$, and let $T(\Delta)=\operatorname{Spec} \mathbb{C}\left[R_{\sigma}\right]$ be the corresponding toric variety. Recall that a toric valuation on a toric variety $X=T(\Delta)$ is determined by a nonzero element in $\sigma \cap N$, and conversely.

Theorem 5.1. Let $v$ be a divisorial valuation over a complex affine toric variety $X=\operatorname{Spec} \mathbb{C}\left[R_{\sigma}\right]$ centered at the origin of $X$. Assume that there is an element $a \in \sigma \cap N \backslash\{0\}$ such that

$$
\widehat{k}_{v}(X)+q(v) \leq \min _{\left\{x_{1}, \ldots, x_{n}\right\}}\left\{\sum\left\langle x_{i}, a\right\rangle\right\},
$$

where the minimum is taken over linearly independent subsets $\left\{x_{1}, \ldots, x_{n}\right\} \subset$ $R_{\sigma}$ of cardinality $n=\operatorname{dim} X$, and that

$$
v\left(u_{j}\right) \geq\left\langle u_{j}, a\right\rangle \quad \text { for all } j
$$

for some set of monomial generators $\left\{u_{1}, \ldots, u_{r}\right\}$ of $R_{\sigma}$. Then $v$ coincides with the toric valuation $\operatorname{val}_{a}$ determined by $a, q(v)=\max \{k \in \mathbb{N} \mid a \in k N\}$, and equality holds in (5) and (6) 
The proof for the singular toric case is similar, but we need to fix a couple of properties first. From now on, suppose that $X=T(\Delta)$ (notation as above). Fix a nonzero element $a \in \sigma \cap N$, and let $\operatorname{val}_{a}$ be the associated toric valuation.

Lemma 5.2. With the above notation, we have

$$
\widehat{k}_{\mathrm{val}_{a}}(X)=\min _{\left\{x_{1}, \ldots, x_{n}\right\}}\left(\sum\left\langle x_{i}, a\right\rangle-q(a)\right),
$$

where $q(a)=\max \{k \in \mathbb{N} \mid a \in k N\}$, and the minimum is taken over the set of linearly independent subsets $\left\{x_{1}, \ldots, x_{n}\right\}$ of cardinality $n$ in $R_{\sigma}$.

Proof. By replacing $a$ by its primitive element $a / q(a)$, we can assume that $q(a)=1$. We observe that $\wedge^{n} \Omega_{X}$ is generated by the torus-invariant forms

$$
d x_{1} \wedge \cdots \wedge d x_{n}
$$

as $\left\{x_{1}, \ldots, x_{n}\right\}$ ranges among linearly independent subsets of cardinality $n$ in $\sigma^{\vee} \cap M$.

Take a toric resolution of singularities $f: Y \rightarrow X$ factoring both through the toric blowup $g: \mathrm{Bl}_{a}(X) \rightarrow X$ determined by $a$, and through the Nash blowup $\nu: \widehat{X} \rightarrow X$. Let $E \subset Y$ be the prime divisor that is the proper transform of the exceptional divisor of $g$, and fix toric invariant coordinates $\left(y_{1}, \ldots, y_{n}\right)$ in a toric affine open set of $Y$ intersecting $E$. Note that $E$ is defined by the vanishing of one of these $y_{j}$.

Note that $\wedge^{n} \Omega_{Y} \cong \mathcal{O}_{Y}\left(K_{Y}\right)$ is generated (in this open set) by the form

$$
d y_{1} \wedge \cdots \wedge d y_{n}
$$

On the other hand, the image of the map $d f: f^{*} \wedge^{n} \Omega_{X} \rightarrow \wedge^{n} \Omega_{Y}$ is determined, in this open set, by

$$
d x_{1} \wedge \cdots \wedge d x_{n}=\left(x_{1} \ldots x_{n}\right) \cdot \frac{d y_{1}}{y_{1}} \wedge \cdots \wedge \frac{d y_{n}}{y_{n}} \quad \text { up to unit }
$$

as $\left\{x_{1}, \ldots, x_{n}\right\}$ ranges as above. The statement follows by the definition of $\widehat{K}_{Y / X}$ as the divisor determined by the image of $d f$.

The next step is to understand the maximal divisorial set associated to the toric valuation $\operatorname{val}_{a}$. We fix nonconstant monomial generators $u_{1}, \ldots, u_{r}$ of $R_{\sigma}$, and let $a_{j}:=\left\langle u_{j}, a\right\rangle$.

Lemma 5.3. The maximal divisorial set associated with $\operatorname{val}_{a}$ is equal to

$$
W\left(\operatorname{val}_{a}\right)=\left\{\gamma \in X_{\infty} \mid \operatorname{ord}_{\gamma}\left(u_{j}\right) \geq a_{j}\right\}
$$


Proof. The inclusion $\subset$ is trivial. We prove the opposite inclusion $\supset$. Let $T \cong\left(\mathbb{C}^{*}\right)^{n}$ be the torus acting on $X$. For every $m \geq 1$ we have a commutative diagram

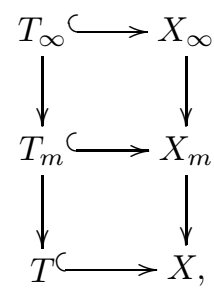

where $T_{m} \cong \mathbb{C}^{n m} \times\left(\mathbb{C}^{*}\right)^{n}$ acts on $X_{m}$ ([I1, Proposition 2.6]), and $T_{\infty}$ is the proscheme obtained as the inverse limit of the projective system $\left\{T_{m} \rightarrow T_{m-1}\right\}$.

For every face $\tau \preceq \sigma$ we define

$$
X_{\infty}(\tau):=\left\{\gamma \in X_{\infty} \mid \gamma(\eta) \in \operatorname{orb}(\tau)\right\},
$$

in particular for $\tau=\{0\}$

$$
X_{\infty}(0):=\left\{\gamma \in X_{\infty} \mid \gamma(\eta) \in T\right\}
$$

where $\eta$ denotes the generic point of $\operatorname{Spec} \mathbb{C}(\gamma)[[t]]$ and $\mathbb{C}(\gamma)$ is the residue field of $\gamma \in X_{\infty}$. We obtain a stratification

$$
X_{\infty}=\bigsqcup_{\tau \preceq \sigma} X_{\infty}(\tau) .
$$

Observe that every arc $\gamma: \mathbb{C}\left[R_{\sigma}\right] \rightarrow \mathbb{C}(\gamma)[[t]]$ determines a semigroup map

$$
\operatorname{ord}_{\gamma}: R_{\sigma} \rightarrow \mathbb{Z}_{\geq 0} \cup\{\infty\} \text {. }
$$

If $\gamma \in X_{\infty}(0)$, then we actually have ord ${ }_{\gamma}: R_{\sigma} \rightarrow \mathbb{Z}_{\geq 0}$, and this extends to a linear map $M \rightarrow \mathbb{Z}$. So $\gamma$ naturally determines an element $a_{\gamma} \in N$, and since $\operatorname{ord}_{\gamma}$ is nonnegative on $R_{\sigma}$, we have $a_{\gamma} \in \sigma$.

Now we stratify $X_{\infty}(0)$ accordingly to the position of $a_{\gamma}$ (we treat the other strata $X(\tau)$, for $0 \neq \tau \preceq \sigma$, by induction on dimension) as follows. For every $a \in \sigma \cap N \backslash\{0\}$ we define

$$
T_{\infty}(a)=\left\{\gamma \in X_{\infty}(0) \mid a_{\gamma}=a\right\} .
$$

Then $T_{\infty}(a)$ is a $T_{\infty}$-orbit and $\overline{T_{\infty}(a)}=W\left(\operatorname{val}_{a}\right)$.

By [I1, Proposition 4.8], for every two $a, a^{\prime} \in(\sigma \cap N) \backslash\{0\}$ we also have

$$
\overline{T_{\infty}\left(a^{\prime}\right)} \subseteq \overline{T_{\infty}(a)} \Longleftrightarrow a^{\prime}-\left.a \in \sigma \cap N \quad \Longleftrightarrow \quad \operatorname{val}_{a^{\prime}}\right|_{R_{\sigma}} \geq\left.\operatorname{val}_{a}\right|_{R_{\sigma}} .
$$


Now take an arbitrary $\gamma \in X_{\infty}(0) \backslash W\left(\operatorname{val}_{a}\right)$. Then $\gamma \in T_{\infty}(b)$ for some $b \in(\sigma \cap N) \backslash\{0\}$ for which $b-a \notin \sigma \cap N$, hence we have $\operatorname{ord}_{\gamma}\left(u_{j}\right)<a_{j}$ for some $u_{j}$. The statement follows by iterating the argument on the different pieces $X(\tau)$ of the stratification of $X$ by using [I1, Theorem 4.15].

Proof of Theorem 5.1. The proof goes along the same lines of the one of Theorem 0.3. By Lemma 5.3 and (6), we have $W(v) \subseteq W\left(\operatorname{val}_{a}\right)$. The conclusion follows by comparing codimension, which are determined respectively by (5) and Lemma 5.2.

\section{References}

[B] V. V. Batyrev, Non-Archimedean integrals and stringy Euler numbers of log-terminal pairs, J. Eur. Math. Soc. (JEMS) 1 (1999), no. 1, 5-33.

[CGP] V. Cossart, C. Galindo and O. Piltant, Un exemple effectif de gradué non noethérien associé à une valuation divisorielle, Ann. Inst. Fourier (Grenoble) 50 (2000), no. 1, $105-112$.

[DL] J. Denef and F. Loeser, Germs of arcs on singular algebraic varieties and motivic integration, Invent. Math. 135 (1999), no. 1, 201-232.

[ELM] L. Ein, R. Lazarsfeld and M. Mustaţă, Contact loci in arc spaces, Compos. Math. 140 (2004), no. 5, 1229-1244.

[EM1] L. Ein and M. Mustață, Inversion of adjunction for local complete intersection varieties, Amer. J. Math. 126 (2004), no. 6, 1355-1365.

[EM2] Jet schemes and singularities. Math. AG/0612862v1.

[F] W. Fulton, Introduction to toric varieties, Ann. of Math. Stud., 131, Princeton Univ. Press, Princeton, NJ, 1993.

[K] M. Kontsevich, Motivic integration, Lecture at Orsay, 1995.

[I1] S. Ishii, The arc space of a toric variety, J. Algebra 278 (2004), no. 2, 666-683.

[I2] _ Arcs, valuations and the Nash map, J. Reine Angew. Math. 588 (2005), $71-92$.

[I3] _ Maximal divisorial sets in arc spaces, preprint, to appear in the Proceeding of Algebraic Geometry in East Asia 2005.

[ML] S. MacLane, A construction for absolute values in polynomial rings, Trans. Amer. Math. Soc. 40 (1936), no. 3, 363-395.

[M1] M. Mustaţă, Jet schemes of locally complete intersection canonical singularities, Invent. Math. 145 (2001), no. 3, 397-424.

[M2] Singularities of pairs via jet schemes, J. Amer. Math. Soc. 15 (2002), no. 3, 599-615 (electronic).

[N] J. F. Nash, Jr., Arc structure of singularities, Duke Math. J. 81 (1995), no. 1, 31-38 (1996). 